Proceedings

\title{
Investigations on Long-Range AFM Scans Using a Nanofabrication Machine (NFM-100) ${ }^{+}$
}

\author{
Jaqueline Stauffenberg 1,*, Ingo Ortlepp ${ }^{1}$, Christoph Reuter ${ }^{2}$, Mathias Holz ${ }^{3}$, Denis Dontsov ${ }^{4}$, \\ Christoph Schäffel ${ }^{5}$, Steffen Strehle ${ }^{2}$, Jens-Peter Zöllner ${ }^{6}$, Ivo W. Rangelow ${ }^{7}$ \\ and Eberhard Manske ${ }^{1}$ \\ 1 Production and Precision Measurement Technology, Technische Universität Ilmenau, Gustav-Kirchhoff-Straße \\ 1, 98693 Ilmenau, Germany; ingo.ortlepp@tu-ilmenau.de (I.O.); eberhard.manske@tu-ilmenau.de (E.M.) \\ 2 Microsystems Technology Group, Technische Universität Ilmenau, Max-Planck-Ring 12, 98693 Ilmenau, \\ Germany; christoph.reuter@tu-ilmenau.de (C.R.); steffen.strehle@tu-ilmenau.de (S.S.) \\ 3 Nano Analytik GmbH, Ehrenbergstr. 1, 98693 Ilmenau, Germany; m.holz@nanoanalytik.de \\ 4 SIOS Meßtechnik GmbH, Am Vogelherd 46, 98693 Ilmenau, Germany; dontsov@sios.de \\ 5 Institut für Mikroelektronik- und Mechatronik-Systeme gemeinnützige GmbH (IMMS gGmbH), \\ Ehrenbergstraße 27, 98693 Ilmenau, Germany; christoph.schaeffel@imms.de \\ 6 Micro- and nanoelectronic Systems Group, Technische Universität Ilmenau, Gustav-Kirchhoff-Straße 1, \\ 98693 Ilmenau, Germany; jens-peter.zoellner@tu-ilmenau.de \\ 7 Nanoscale Systems Group, Technische Universität Ilmenau, Gustav-Kirchhoff-Straße 1, 98693 Ilmenau, \\ Germany; ivo.rangelow@tu-ilmenau.de \\ * Correspondence: jaqueline.stauffenberg@tu-ilmenau.de \\ + Presented at the 4th International Conference nanoFIS 2020-Functional Integrated nano Systems, Graz, \\ Austria, 2-4 November 2020.
}

Published: 30 December 2020

\begin{abstract}
The focus of this work lies on investigations on a new Nano Fabrication Machine (NFM100) with a mounted atomic force microscope (AFM). This installed tip-based measuring system uses self-sensing and self-actuated microcantilevers, which can be used especially for field-emission scanning probe lithography (FESPL). The NFM-100 has a positioning range of $\varnothing 100 \mathrm{~mm}$, which offers, in combination with the tip-based measuring system, the possibility to analyse structures over long ranges. Using different gratings, the accuracy and the reproducibility of the NFM-100 and the AFM-system will be shown.
\end{abstract}

Keywords: nanopositioning; nanometrology; AFM; self-sensing microcantilevers

\section{Introduction}

Most of the tip-based systems are using standard Atomic Force Microscope equipment and work with high resolution and reproducibility in small areas of several micrometers [1]. For this purpose, it becomes more and more important to overcome these limitations and to fabricate and analyse structures over long ranges. In this contribution, the focus lies on the investigations on the new Nano Fabrication Machine 100 (NFM-100), which has a positioning range of $\varnothing 100 \mathrm{~mm}$ and poses an important platform for basic research in the field of tip- and laser-based nanomeasuring and nanofabrication. To investigate new tip-based measuring systems in combination with the NFM-100 in large working areas, an Atomic Force Microscope (AFM) is installed. 


\section{Design of the Nano Fabrication Machine 100 and the Mounted AFM System}

Based on the experiences with the Nanomeasuring Machine 1 (NMM-1) and the Nanopositioning and Nanomeasuring Machine (NPMM-200), the NFM-100 was developed at the Technische Universität Ilmenau, Institute for Process Measurement and Sensor Technology and IMMS gGmbH. In collaboration with SIOS Meßtechnik GmbH, IMMS gGmbH and nanoanalytik $\mathrm{GmbH}$, the combination of the NFM-100 and its tip-based measuring system was realized. For high accuracy positioning in the $\mathrm{x}$ - and $\mathrm{y}$-directions, this machine uses a planar driving system. The slider is moved by three linear actuators, which consist of flat coils and a magnet array each [2]. These actuators are arranged in a single plane and have an angle of $120^{\circ}$ between each other. The position of the slider can be determined by three fibre-coupled laser interferometers.

Additionally, an atomic force microscope is installed, which uses self-sensing and self-actuated microcantilevers. These microcantilevers use a wheatstone bridge of four piezoresistors to detect the deflection and a thermomechanical actuator, which uses the bimorph effect for oscillation [3].

\section{AFM Scan Results over Long Range}

To show the performance of the mounted AFM-System and the NFM-100, a step grating is used. The grating has a pitch size of $3 \mu \mathrm{m}$ and depth of approximately $560 \mathrm{~nm}$. During the measurement, the microcantilever is in a fixed position in the $\mathrm{x}$ - and $\mathrm{y}$-directions, while the z-position is controlled by the piezo-scanning unit. The movement in the $\mathrm{x}$ - and $\mathrm{y}$-directions is realized by the NFM-100. As an example, a line scan with a length of $3 \mathrm{~mm}$ was performed with a velocity of $1 \mu \mathrm{m} / \mathrm{s}$ (see Figure 1). In principle, it is possible to analyse samples over an area up to $\varnothing 100 \mathrm{~mm}$. A detail of the result of this measured line scan can be found in Figure 1.

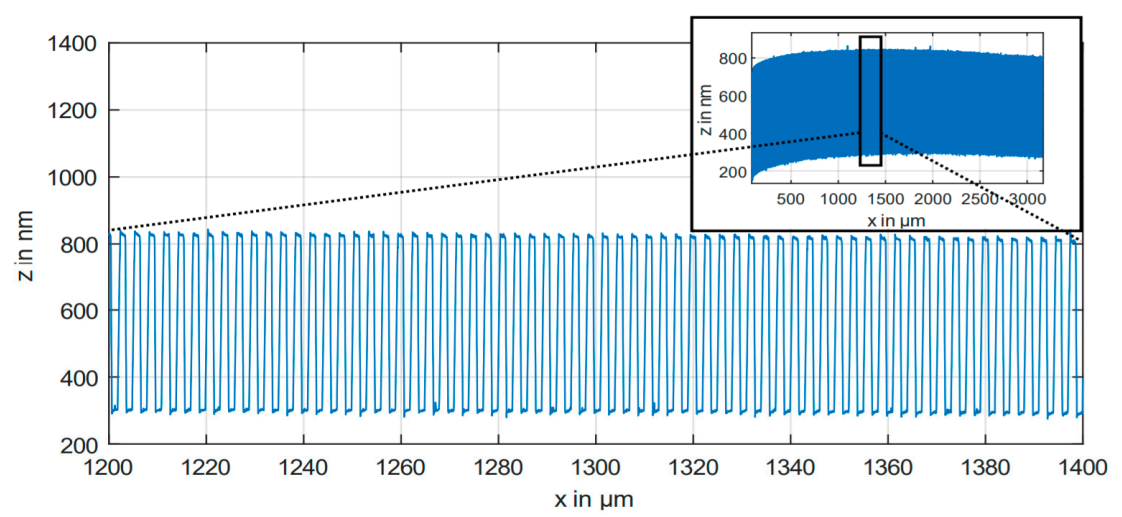

Figure 1. Line scan of $3 \mathrm{~mm}$ with a count of 1000 pitches and section (200 $\mu \mathrm{m}$ in length) of the line scan of a calibration sample with the NFM-100 and its mounted AFM system over a range of $3 \mathrm{~mm}$.

Author Contributions: conceptualization, E.M.; supervision, E.M., I.O., J.-P.Z. and S.S.; investigation of the planar driving system, D.D. and C.S.; investigation of the AFM system, I.W.R., M.H. and C.R.; software and data readout, C.R.; investigation and realization of the measurements, J.S.; evaluation, J.S. and I.O.; writing-original draft preparation and editing, J.S.; writing-review, E.M. and I.O. All authors have read and agreed to the published version of the manuscript.

Funding: This research was funded by Deutsche Forschungsgemeinschaft, INST 273/75-1 FUGG and TMWWDG/EFRE 2018 HSB 014. The APC was funded by Technische Universität Ilmenau.

Acknowledgments: The authors acknowledge the support of the Deutsche Forschungsgemeinschaft in the scope of the Research Training Group “Tip- and Laserbased 3D-Nanofabrication in extended macroscopic working areas" (GRK 2182) at the Technische Universität Ilmenau, Germany.

Conflicts of Interest: The authors declare no conflicts of interest. 


\section{References}

1. Ortlepp, I.; Kühnel, M.; Hofmann, M.; Weidenfeller, L.; Kirchner, J.; Supreeti, S.; Mastylo, R.; Holz, M.; Michels, T.; Füßl, R.; et al. Tip- and Laser-Based Nanofabrication up to $100 \mathrm{~mm}$ with sub-Nanometer Precision; SPIE Advanced Lithography: San Jose, CA, USA, 2020; p. 11324-8.

2. Hesse, S.; Schäffel, C.; Mohr, H.U.; Katschmann, M.; Büchner, H.J. Design and performance of an interferometric controlled planar nanopositioning system. Meas. Sci. Technol. 2012, 23, 074011.

3. Rangelow, I.W.; Ivanov, T.; Hudek, T.P.; Fortagne, O. Device and Method for Mask-Less AFM Microlithography. U.S. Patent 7, 141,808, 2005.

Publisher's Note: MDPI stays neutral with regard to jurisdictional claims in published maps and institutional affiliations.

(C) 2020 by the authors. Licensee MDPI, Basel, Switzerland. This article is an open access article distributed under the terms and conditions of the Creative Commons Attribution (CC BY) license (http://creativecommons.org/licenses/by/4.0/). 